\title{
Classical canonical transformation theory as a tool to describe multidimensional tunnelling in reactive scattering. Hopping method revisited and collinear $\mathrm{H}+\mathrm{H}_{2}$ exchange reaction near the classical threshold
}

\author{
Gennady V. Mil'nikov and António J. C. Varandas* \\ Departamento de Química, Universidade de Coimbra, 3049 Coimbra Codex, Portugal
}

Received 3rd November 1998, Accepted 4th January 1999

\begin{abstract}
Classical canonical perturbation theory is applied in the vicinity of the saddle point for a chemical reaction. This is done by applying successive canonical transformations in the scope of the Gustavson-Birkhoff approach. It is shown that the calculated approximate classical integrals of motion can be used to describe classically forbidden tunnelling processes. They are also organically embedded into a hopping method to incorporate tunnelling effects into classical trajectory simulations of chemical reactions. The applicability of the proposed scheme is demonstrated for the collinear $\mathrm{H}+\mathrm{H}_{2}$ exchange reaction using the double many-body expansion potential energy surface.
\end{abstract}

\section{Introduction}

Tunnelling is an ubiquitous physical phenomenon which plays an essential role in phase transitions, ${ }^{1,2}$ quantum field theory, ${ }^{3-5}$ nuclear physics, ${ }^{6}$ and solid state physics. ${ }^{7}$ In chemical physics, molecular dissociations and interconversion as well as light-atom transfer reactions with activation energy are processes where it is also known to be important. ${ }^{8-10}$

Tunnelling is generally understood as a purely quantum process when the system under consideration passes through the potential barrier from one classically acceptable region to another. The quantum probability for such classically forbidden process is nonzero although the wave function (or probability density) may be exponentially damped. Thus, the full information about the tunnelling process must in principle be obtained by solving the Schrödinger equation with appropriate boundary conditions. In practice, however, the exact quantum solution is often unaffordable and one has to resort to miscellaneous approximations. Since in the tunnelling region the wave function may change in absolute value by orders of magnitude, the usual perturbative methods are inappropriate for this purpose and non-perturbative semiclassical (WKB) approximations have been of primary interest for treating such tunnelling phenomena.

In the scope of the WKB approach one has to deal with the Hamilton-Jacobi (HJ) equation, and only in a few cases (such as 1D systems or others which can be recast as 1D) can its solution be easily found. In the multidimensional case, the HJ equation is a partial differential equation, and to find its solution is almost as difficult as to solve exactly the quantum problem. There are three basic reasons why multidimensionality plays a crucial role in the application of the WKB method to tunnelling problems. First, the solution $W(\mathbf{r})$ of the HJ equation is generally determined with the accuracy of some arbitrary function in the $\mathrm{N}$-dimensional coordinate space, and cannot be considered without reference to some specific boundary conditions. These must be prescribed on some region of the $(N-1)$-dimensional subspace (initial Lagrange manifold). Only for $N=1$ does the problem of boundary conditions become trivial and can be reduced to a turning point. Second, for tunnelling problems, $W(\mathbf{r})$ is generally complex- valued, which leads to the concept of mixed tunnelling ${ }^{11-13}$ in contrast to that of pure tunnelling when the solution in the classically forbidden region is supposed to be purely imaginary. It was pointed out elsewhere ${ }^{11,13}$ that, unlike the $1 \mathrm{D}$ case, pure tunnelling is not always adequate in the multidimensional case. At the same time, for mixed tunnelling, no simple analog of the method of characteristics is available for the classically unacceptable region. Third, the existence of several branches of $W(\mathbf{r})$ leads to another purely quantum effect, i.e., interference, which does not appear in the $1 \mathrm{D}$ case. Its full study is related with the accurate investigation of Stokes phenomena which is handicapped by the absence of an analytical solution for the $\mathrm{HJ}$ equation in the general case.

The numerous approximate quasiclassical theories which either reduce the dimensionality of the problem or prescribe some tunnelling path in configuration space (escape path, tunnelling mode, etc.) have overlooked so far the abovementioned questions. Alternative approaches resort to the saddle point or stationary phase approximation in the path integral formalism of quantum mechanics and statistics. In this way, truly multidimensional results (such as instanton theory, ${ }^{14,15}$ path decomposition expansion, ${ }^{16}$ and $S$-matrix theory ${ }^{17,18}$ ) have been obtained. It has also been shown that the instanton theory can be reformulated in terms of the HJ equation for the inverted potential with specific boundary conditions near its top. ${ }^{19,20}$ However, it is not relevant for scattering problems. Moreover, instanton-like results can be applied only in the case of pure tunnelling and this strict limitation relates actually to any multidimensional theory that deals with the most probable tunnelling path in real configuration space. Mathematically, such a limitation is due to the fact that the complex-valued solution of the HJ equation is described in terms of not one but two coupled sets of EulerLagrange equations which are not equivalent to a single set of ordinary differential equations. ${ }^{13}$ In collisional problems, complex classical trajectories have been used to calculate $S$ matrix elements for classically forbidden processes. ${ }^{21,22}$ Thus, semiclassical $S$-matrix theory is formally free from the abovementioned drawback, although the extension of the path integral formalism to complex phase space and its relation with complex classical mechanics needs a more rigorous mathe- 
matical foundation. Besides, it requires the proper analytical behavior of the potential energy surface in complex coordinate space, which cannot be warranted for most existing models.

From the pragmatic point of view, the above-mentioned "exact" theories are rather difficult to implement for dimensionalities higher than two. Thus, they are often impractical even for the simplest chemical objects which allow an exact quantum mechanical treatment. For more complicated polyatomic systems, the only computationally affordable tool in reaction dynamics has been so far classical trajectory simulations. These are known to provide good average results for reactivity even in the least favorable case of the $\mathrm{H}+\mathrm{H}_{2}$ reaction, but fail to describe purely quantum effects such as tunnelling and zero-point energy conservation. Thus, it is hardly desirable to develop a method for classical trajectory simulations which is applicable to any dimensionality and incorporates tunnelling in it. In this sense, the trajectory hopping method looks a promising route to describe tunnelling effects. ${ }^{23,24}$ According to its simplest version, the classical trajectories are allowed to jump through the classically forbidden region with some prescribed probability which must be calculated for every trajectory. Although this is similar in spirit to the trajectory surface hopping method for treating nonadiabatic reactions, ${ }^{25}$ the justification for such a strategy and its physical interpretation is less clear. Indeed, for a classically acceptable region, a single characteristic of the $\mathrm{HJ}$ equation (classical trajectory) bears no physical meaning. Only a family of characteristics conforming with the appropriate boundary conditions provides a WKB solution. Clearly, the full WKB analysis of tunnelling must appeal to the whole family of characteristics, or, generally speaking, tunnelling cannot be incorporated into a single trajectory. Thus, a single trajectory cannot also be continued in the forbidden region by means of complex time or any other single complex parameter (unlike the one-dimensional tunnelling where the complex time method has been shown to recover the usual WKB results ${ }^{26}$ ).

The questions we address are therefore: (i) whether there is any possibility to justify the intuitive hopping recipe for multidimensional tunnelling, and (ii) how to calculate the tunnelling hopping probability. Partly, the ideas which substantiate the current work have already been outlined in a previous publication, ${ }^{27}$ where we have proposed a new procedure for calculating the hopping probability. In fact, the key problems of multidimensional tunnelling delineated in the previous paragraphs have not been addressed in our previous paper while the computational strategy itself was mostly based on an intuitive analogy with the separable case. In this paper we cover such a gap, and examine our earlier approach from a more rigorous position.

To elucidate the main ideas, we first note that there are basically two ways to calculate complex-valued $W(\mathbf{r})$ solutions in the general case. The first is through the direct numerical solution of the HJ equation, which can be done by Huygenstype construction $;^{13,28}$ this consists of a successive evaluation of equipotential surfaces for $\operatorname{Re} W(\mathbf{r})$ and $\operatorname{Im} W(\mathbf{r})$. The solution is joined smoothly onto the classically allowed region, providing the analytical continuation of the real-valued $W(\mathbf{r})$. The second approach consists of obtaining first a closed algebraic form for $W(\mathbf{r})$ in the classically allowed region, which is then assumed to be valid in the forbidden region. This concept has recently been implemented by Takada ${ }^{29}$ who has used classical canonical perturbation theory to get an approximation of quantized tori which could then be analytically continued at least in the neighboring classical forbidden area. Since in any tunnelling problem a forbidden region always separates two allowed regions, we find it more natural to invert in some sense Takada's approach. Thus, we look for some approximate form of $W(\mathbf{r})$ which is valid in the tunnelling region, and also in the neighboring classically acceptable regions. These must be used to determine the boundary conditions appropriate for the given problem. Although such a consideration is relevant for any kind of tunnelling phenomena, only scattering processes are mostly addressed in this paper. In this case, the boundary conditions near the frontier of the classically allowed regions can be easily calculated through classical trajectory simulations. We apply classical canonical transformation ${ }^{30-32}$ within the Gustavson-Birkhoff formalism to reduce the initial Hamiltonian to its simplest possible form, and to calculate all the classical integrals of motion. Such a reduction is chosen to be valid in the vicinity of the saddle point of the potential energy surface. We shall find formally correct expressions for $W(\mathbf{r})$ and the reaction probability, and show that the calculated integrals of motion can be used within the hopping method. The latter will be illustrated for the collinear $\mathrm{H}+\mathrm{H}_{2}$ exchange reaction at collision energies near the classical threshold using the double many-body expansion (DMBE) potential energy surface. ${ }^{33}$ Interference effects cannot be described in the scope of the current hopping method, and we shall not address to this issue any further in the present work.

The paper is organized as follows. Section 2 presents the flux formulation for the tunnelling collision probability in the semiclassical approximation. In Section 3 we apply the classical perturbation theory to get the approximate solution of the $\mathrm{HJ}$ equation, and formulate the numerical strategy for the hopping method. Section 4 outlines the details of the computational strategy and presents the main results which have been obtained for the title reaction. Concluding remarks are in Section 5. For completness some aspects of the classical canonical perturbation theory used in the present work are summarized in the Appendix.

\section{Tunnelling probability in the WKB approximation}

We consider the simplest case of a collinear $\mathrm{A}+\mathrm{BC} \rightarrow \mathrm{AB}+\mathrm{C}$ exchange reaction with a total energy below the activation potential barrier, and denote the initial (final) arrangement channel by $i(f)$. The rather obvious generalization of notations makes the following results also applicable to the more general case. Our aim is to express the reaction probability in terms of the WKB solution of Schrödinger's equation $\Psi_{v_{i}}(\mathbf{q})$, which corresponds to an incoming wave of unit flux in the initial arrangement channel $i$. We start with a brief summary of the method of characteristics which supplies the WKB solution in the classically allowed region.

Let us introduce the compact notation $\mathbf{q}$ and $\mathbf{p}$ for coordinates $\left(q_{1}, q_{2}\right)$ and their conjugate momenta $\left(p_{1}, p_{2}\right)$, and consider the one-parameter family of characteristics $[\mathbf{q}(\alpha, t)$, $\mathbf{p}(\alpha, t)]$. These are classical trajectories which satisfy Hamilton's equation of motion with initial conditions

$$
\begin{aligned}
& \mathbf{q}(\alpha, t=0)=\mathbf{q}_{0}(\alpha) \\
& \mathbf{p}(\alpha, t=0)=\mathbf{p}_{0}(\alpha)
\end{aligned}
$$

where $\left[\mathbf{q}_{0}(\alpha), \mathbf{p}_{0}(\alpha)\right]$ defines the initial Lagrange manifold. Next we determine the single valued function $W(\alpha, t)$

$$
W(\alpha, t)=W_{0}(\alpha)+\int_{\alpha, 0}^{\alpha, t} \mathbf{p}(\alpha, \tau) \mathrm{d} \mathbf{q}(\alpha, \tau)
$$

where the integral is taken along the characteristics at a fixed value of the parameter $\alpha$, as explicitly indicated in eqn. (2). To finally get $W$ as a function of the coordinates, $W_{0}(\alpha)$ must be defined on the initial Lagrange manifold as

$$
W_{0}(\alpha)=\int^{\alpha} \mathbf{p}_{0}\left(\alpha^{\prime}\right) \mathrm{d} \mathbf{q}_{0}\left(\alpha^{\prime}\right)
$$


The WKB wave function is then given locally by ${ }^{34,35}$

$$
\Psi(\mathbf{q})=\left.\sum_{v} \sqrt{f\left(\alpha_{v}\right)}\left[\frac{\mathscr{D}\left(q_{1}, q_{2}\right)}{\mathscr{D}(\alpha, t)}\right]^{-1 / 2}\right|_{v} \exp \left(\frac{\mathrm{i} W_{v}}{\hbar}\right)
$$

where $f(\alpha)$ is a function which depends on the choice of the parameter $\alpha$, which will be specified below; the term in square brackets denotes the Jacobian of the $\left(q_{1}, q_{2}\right) \leftrightarrow(\alpha, t)$ transformation. The lower integration limit in eqn. (3) is not essential and only affects the constant phase of the wave function. Although $W$ is a single-valued function of $(\alpha, t)$ it becomes multivalued in the full configuration space, which explains the appearance of the branch index $v$ in eqn. (4). The different branches $W_{v}(\mathbf{q})$ supply the solutions of the HJ equation subject to the boundary condition

$$
W_{v}\left(\mathbf{q}_{0}\right)=W_{0}\left(\alpha_{v}\right)
$$

while the branching lines are caustics where the mapping $\left(q_{1}, q_{2}\right) \leftrightarrow(\alpha, t)$ becomes singular

$$
\frac{\mathscr{D}\left(q_{1}, q_{2}\right)}{\mathscr{D}(\alpha, t)}=0
$$

and hence the WKB approximation breaks down. Note that the problem of constructing a global WKB solution has been solved by Maslov and co-workers ${ }^{34}$ using a mixed coordinate-momentum representation in the vicinity of the singularities and successive matching of local solutions. The final result has the same form as in eqn. (4) except for the extra phase $-\mathrm{i} \pi \mu / 2$ where $\mu$ is the Morse index of the characteristic. $^{34}$ Since the effect of interferences is left out in the present analysis we will not indicate this extra phase explicitly and omit also the branch index hereafter. Further details concerning the construction of the global WKB solution can be found in ref. 34.

It is now convenient to rewrite eqn. (4) in a different form. Following Wilkinson, ${ }^{36}$ we make a local orthogonal transformation to the new coordinate system $(\eta, \xi)$, such that $p_{\xi}=0$. The WKB wave function assumes then the form

$$
\Psi(\mathbf{q})=\sqrt{f(\alpha)}\left[v \frac{\mathrm{d} \xi}{\mathrm{d} \alpha}\right]^{-1 / 2} \exp \left(\frac{\mathrm{i} W}{\hbar}\right)
$$

where $v=\partial n / \partial t$ is the velocity along the characteristics. Note that for the general multidimensional case, the factor $\mathrm{d} \xi / \mathrm{d} \alpha$ in eqn. (7) is replaced by the Jacobian of the mapping via rays. ${ }^{37}$

To describe tunnelling we need to consider $\Psi\left(\mathbf{q}^{\prime}\right)$ in regions where the equation

$$
\mathbf{q}^{\prime}=\mathbf{q}(\alpha, t)
$$

has no real-valued roots, and hence the WKB wave function must be expressed in terms of complex classical mechanics. This is equivalent to the analytical continuation of $W(\mathbf{q})$ as formulated by Wilkinson. ${ }^{36}$ For complex-valued $(\alpha, t)$, or equally in complex coordinate space, the WKB wave function still preserves its form in eqn. (7). To show this we closely follow the derivation given by Takada ${ }^{29}$ [with minor changes owing to the different representation of the WKB wave function in eqn. (7)], and utilize a decomposition approach based on Green's theorem. For simplicity we take $\hbar=M=1$ and apply Green's theorem

$$
\Psi\left(\mathbf{q}^{\prime}\right)=-\frac{1}{2} \int_{\Sigma} \mathrm{d} \mathbf{q}\left[G\left(\mathbf{q}^{\prime}, \mathbf{q}\right) \frac{\mathrm{d} \Psi(\mathbf{q})}{\mathrm{d} \mathbf{q}}-\frac{\mathrm{d} G\left(\mathbf{q}^{\prime}, \mathbf{q}\right)}{\mathrm{d} \mathbf{q}} \Psi(\mathbf{q})\right]
$$

with the semiclassical Green's function being given by ${ }^{38}$

$$
G\left(\mathbf{q}^{\prime}, \mathbf{q}\right)=(2 \pi \mathrm{i})^{-1 / 2}\left(\frac{1}{p_{\eta} p_{\eta}^{\prime}} \frac{\partial^{2} W^{\prime}}{\partial \xi \partial \xi^{\prime}}\right)^{1 / 2} \exp \left(\mathrm{i} W^{\prime}\right)
$$

where $W^{\prime}\left(\mathbf{q}, \mathbf{q}^{\prime}\right)$ is the action integral along the classical trajectory (generally complex) connecting $\mathbf{q}$ and $\mathbf{q}^{\prime}$. We locate the surface $\Sigma$ (line in the 2D case) in the classically allowed region and perform the integration in eqn. (9) using the stationary phase approximation. The direct calculation, similar to that in ref. 29 , gives

$$
\begin{aligned}
\Psi\left(\mathbf{q}^{\prime}\right)= & \sqrt{f\left(\alpha^{*}\right)}\left[p_{\eta}^{\prime} \frac{\mathrm{d} \xi}{\mathrm{d} \alpha}\right]_{*}^{-1 / 2} \\
& \times\left[-\left(\frac{\partial^{2} W^{\prime}}{\partial \xi \partial \xi^{\prime}}\right)^{-1} \frac{\partial^{2}}{\partial \xi^{2}}\left(W+W^{\prime}\right)\right]_{*}^{-1 / 2} \\
& \times \exp \left[\frac{\mathrm{i}\left(W+W^{\prime}\right)_{*}}{\hbar}\right]
\end{aligned}
$$

where the asterisk indicates that the variables are evaluated at the critical point $\mathbf{q}^{*}$ which is a solution of the stationary phase condition

$$
\frac{\partial}{\partial s}\left[W(\mathbf{q})+W^{\prime}\left(\mathbf{q}, \mathbf{q}^{\prime}\right)\right]=0
$$

and $s$ is the coordinate along $\Sigma$. It is rather easy to prove that eqn. (11) concurs with eqn. (7). Indeed, the secondary phase condition together with the energy conservation law may be shown to lead to the more general equation

$$
\frac{\partial}{\partial \mathbf{q}}\left[W(\mathbf{q})+W^{\prime}\left(\mathbf{q}, \mathbf{q}^{\prime}\right)\right]=0
$$

which is satisfied along some complex classical trajectory. ${ }^{36}$ Along such a trajectory both $\left(W+W^{\prime}\right)$ and the parameter $\alpha$ are constants, and one has at once $\alpha^{*}=\alpha^{\prime}$ and $W\left(\alpha^{\prime}, t^{\prime}\right)=(W$ $\left.+W^{\prime}\right)_{*}$ where $\left(\alpha^{\prime}, t^{\prime}\right)$ is the solution of eqn. (8). In addition, by using eqn. (13), the expression in square brackets in eqn. (11) is reduced to $\partial \xi^{\prime} / \partial \xi$ which ends the proof. Note that even in the WKB approximation the left-hand side of eqn. (9) is not affected by the choice of $\Sigma$ in the classically acceptable region. The crossing of the family of trajectories $[\mathbf{q}(\alpha, t), \mathbf{p}(\alpha, t)]$ with $\Sigma$ determines in the phase space a one-parameter line $\left[\tilde{\mathbf{q}}_{0}(\alpha)\right.$, $\left.\tilde{\mathbf{p}}_{0}(\alpha)\right]$ which can be regarded itself as the initial Lagrange manifold. Thus, we have some freedom in the choice of initial conditions for the characteristics which can be used for simplifying purposes in the consideration of the tunnelling region.

We proceed now with our main goal to calculate the reaction probability. This can be defined as

$$
N_{v_{i}}=\int_{S} \mathrm{~d} \mathbf{s} j_{v_{i}}(\mathbf{q})
$$

where

$$
j_{v_{i}}(\mathbf{q})=\frac{1}{2 i}\left[\frac{\partial \Psi_{v_{i}}(\mathbf{q})}{\partial \mathbf{q}} \Psi_{v_{i}}^{*}(\mathbf{q})-\frac{\partial \Psi_{v_{i}}^{*}(\mathbf{q})}{\partial \mathbf{q}} \Psi_{v_{i}}(\mathbf{q})\right]
$$

is the current associated with $\Psi_{v_{i}}$. The physical meaning of $N_{v_{i}}$ depends on the choice of the line $S$. Thus, if we take $S$ to be the line $S_{f}$ placed in the asymptotic region of the arrangement $f$, eqn. (15) determines the reaction probability $N_{v_{i} \rightarrow f}$ which is cumulative with respect to the quantum numbers $v_{f}$. To get the WKB approximation for the reaction probability it is more convenient to use the wave function in the form of eqn. (4). Thus, by inserting eqn. (4) in eqn. (15), one obtains

$$
\begin{aligned}
& N_{v_{i}}=\int_{S} \mathrm{~d} s\left|\frac{f(\alpha)}{\mathscr{\mathscr { D } ( q _ { 1 } , q _ { 2 } )}}\right| \operatorname{Re}(\mathbf{n v}) \exp (-2 \operatorname{Im} W) \\
& =\int \mathrm{d} s\left|\frac{\mathrm{d} \alpha}{\mathrm{d} s} f(\alpha)\right| \frac{\operatorname{Re}(\mathbf{n v})}{|(\mathbf{n v})|} \exp (-2 \operatorname{Im} W)
\end{aligned}
$$

where $\boldsymbol{n}$ is the unit vector perpendicular to $S, \boldsymbol{v}$ is a velocity which is proportional to $\nabla W$ and, as usual, the higher order terms in $\hbar$ have been neglected. Eqn. (16) gives the reaction probability in terms of the characteristics and requires the 
solution of eqn. (8) with $q^{\prime} \in S$, which determine both $\alpha(s)$ and $W(\alpha(s), t(s))$.

Let us now return to the definition of the function $f(\alpha)$ which is proportional to the density of trajectories describing the given scattering process. For the scattering wave function $\Psi_{v_{i}}(\mathbf{q})$, this is explicitly written as

$$
f(\alpha) \mathrm{d} \alpha=\frac{\mathrm{d} n}{N}
$$

where $\mathrm{d} n$ is the number of trajectories within the interval $(\alpha \div \alpha+\mathrm{d} \alpha)$ and $N$ is their total number in the family. Indeed let us consider in the asymptotic region $i$ the bunch of $N$ trajectories $\mathbf{q}_{n}(t), n=1, \ldots N$, which describe the incoming wave function, and choose the parameter $\alpha \in(0,2 \pi)$ as a phase variable for the diatomic BC. If we impose a homogeneous distribution of the phase, eqn. (17) gives at once $f(\alpha)=1 / 2 \pi$, which corresponds to the incoming flux $J_{\text {inc }}=1$ as follows from eqn. (16) if one locates the line $S$ in the asymptotic region of the initial arrangement channel. At the same time, using Jacobi coordinates $\left(R_{i}, r_{i}\right)$ for the initial arrangement, the initial conditions for the family of trajectories can be determined in such a way that $R_{i 0}(\alpha)=$ const that it is then possible to show that in the asymptotic region the WKB wave function eqn. (4) assumes the correct form

$$
\Psi\left(R_{i}, r_{i}\right)=\frac{1}{\sqrt{v_{R_{i}}}} \exp \left(-\mathrm{i} P_{R_{i}} R_{i}\right) \Phi_{v_{i}}\left(r_{i}\right)
$$

where $\Phi_{v_{i}}$ is the normalized diatomic wave function

The calculation of the flux in eqn. (16) requires the solution of eqn. (8), which implies, generally, the knowledge of $\mathbf{q}(\alpha, \mathbf{t})$ in complex-valued $(\alpha, t)$ space. However, in the threshold region of a chemical reaction, the vicinity of the saddle point is believed to play the most important role in the description of tunnelling. Thus, we may restrict our consideration to a small area of the complex phase space where the form of the characteristics can be found by use of the classical perturbative approach

\section{Complex-valued characteristics, classical perturbation theory and hopping method}

We begin by specifying $\mathbf{q}$ as the normal coordinates in the saddle point of the potential energy surface, and look for some approximate algebraic form of $W(\mathbf{q})$ at small $\mathbf{q}$. The tunnelling effects are mostly important when $\operatorname{Im} W \leqslant \hbar$, since the tunnelling probability is exponentially damped otherwise. This implies the natural scale $\sim \hbar^{1 / 2}$ for the region $\Omega_{W}$ of configuration space where the approximation for $W(\mathbf{q})$ has to be valid. At the same time we require $\Omega_{W}$ to be sufficiently large to overlap with the classically acceptable regions in the initial and final arrangement channels. Under these conditions we can shift $S_{f}$ from the asymptotic region of the final arrangement channel to $\Omega_{W}$, and use classical trajectory simulations to determine the boundary conditions in $\Omega_{W}$ for the solution of the $\mathrm{HJ}$ equation. More explicitly, let us consider again the family parametrized by the phase $\alpha \in(0,2 \pi)$ of the diatomic $\mathrm{BC}$ and prescribe some smooth line $S_{i} \in \Omega_{W}$ which lies totally in the classically allowed area of the initial arrangement channel. For every trajectory $\mathbf{q}(\alpha, t)$ we then determine $t(\alpha)$ as the time when the trajectory first crosses $S_{i}$. This will define the initial Lagrange manifold

$$
\begin{aligned}
& \mathbf{q}_{0}(\alpha)=\mathbf{q}(\alpha, t(\alpha)) \\
& \mathbf{p}_{0}(\alpha)=\mathbf{p}(\alpha, t(\alpha))
\end{aligned}
$$

which uniquely determines the solution of the HJ equation. All the calculations at this stage are purely classical and the functions $\mathbf{q}_{0}(\alpha)$ and $\mathbf{p}_{0}(\alpha)$ can be obtained with any required accuracy. We further assume that the flux through the shifted
$S_{f}$ line can still be identified with the reactive flux. This corresponds to the neglect of "multitunnelling" which, in most of cases, is not a strong limitation of the theory. Thus the problem of calculating the tunnelling probability is reduced to the approximate solution of the HJ equation in $\Omega_{W}$ with given boundary conditions. Within the scope of the method of characteristics we must then consider a small region $\left(\sim \hbar^{1 / 2}\right)$ of the complex phase space where $\mathbf{q}(\alpha, t)$ and $\mathbf{p}(\alpha, t)$ can be found within the formalism of Gustavson-Birkhoff perturbation theory. ${ }^{32}$

\subsection{Characteristics in the tunnelling region}

The details of the implemented perturbative approach can be found elsewhere, ${ }^{27,32}$ and we simply outline here the main steps of the procedure. We use a Taylor series expansion of the potential energy surface in the vicinity of the saddle point, and employ the classical canonical transformation with the second type generator $F_{2}(\mathbf{P}, \mathbf{q})$ defined by

$$
F_{2}(\mathbf{P}, \mathbf{q})=\mathbf{P q}+S(\mathbf{P}, \mathbf{q})
$$

which converts $(\mathbf{q}, \mathbf{p})$ into a set of new $(\mathbf{Q}, \mathbf{P})$ canonical variables

$$
\begin{aligned}
& \mathbf{Q}=\mathbf{q}+\frac{\partial S}{\partial \mathbf{P}} \\
& \mathbf{p}=\mathbf{P}+\frac{\partial S}{\partial \mathbf{q}}
\end{aligned}
$$

The first term in eqn. (20) corresponds to the identity transformation, while $S(\mathbf{P}, \mathbf{q})$ is found in such a way that the new Hamiltonian $\mathscr{H}(\mathbf{P}, \mathbf{Q})$ approximately becomes a polynomial function of $I_{i}$, where

$$
I_{i}\left(P_{i}, Q_{i}\right)=\frac{1}{\left|\omega_{i}\right|}\left(\frac{P_{i}^{2}}{2 M}+\frac{M \omega_{i}^{2} Q_{i}^{2}}{2}\right)
$$

$M$ is the mass (in the case of atom-diatom collisions it is the corresponding reduced mass), and $\omega_{i}$ are the normal frequencies at the saddle point $[S$ is eqn. (20) should not be confused with the notation used before for the surface in the flux integration]. A similar approach is well known for the semiclassical quantization of potential wells and no new concepts are required to calculate $S(\mathbf{P}, \mathbf{q})$ and $\mathscr{H}(\mathbf{P}, \mathbf{Q})$ for the present case when only one of the normal frequencies is imaginary. ${ }^{27}$ In practice the canonical tranformation which is implied in eqn. (20) results from a sequence of transformations

$$
(\mathbf{q}, \mathbf{p}) \stackrel{\boldsymbol{F}_{2}^{(3)\left(\mathbf{P}_{1}, \mathbf{q}\right)} \longrightarrow}{\longrightarrow}\left(\mathbf{Q}_{1}, \mathbf{P}_{1}\right) \stackrel{\boldsymbol{F}_{2}^{(4)\left(\mathbf{P}_{2}, \mathbf{Q}_{1}\right)}}{\longrightarrow}\left(\mathbf{Q}_{2}, \mathbf{P}_{2}\right) \stackrel{\cdots}{\longrightarrow}(\mathbf{Q}, \mathbf{P})
$$

where $F_{2}^{(k)}(k=3,4, \ldots)$ are generators for the canonical transformation which successively remove the anharmonicity of $k$-th order, and have polynomial form in the canonical variables. Within the accuracy of the perturbation expansion, $I_{\mathrm{i}}$ are classical integrals of motion and the characteristics can easily be calculated in terms of the new canonical variables $(\mathbf{Q}, \mathbf{P})$.

Let the coordinate $q_{2}$ correspond to the imaginary normal frequency $\omega_{2}^{2}<0$. In the $(\mathbf{Q}, \mathbf{P})$ variables, Hamilton's equation of motion assumes the form

$$
\begin{gathered}
\dot{Q}_{i}=\frac{\partial H}{\partial I_{i}} \frac{\partial I_{i}}{\partial P_{i}}=\frac{\partial H}{\partial I_{i}} \frac{P_{I}}{M\left|\omega_{i}\right|} \\
\dot{P}_{i}=-\frac{\partial H}{\partial I_{i}} \frac{\partial I_{i}}{\partial Q_{i}}=-\operatorname{sign}\left(\omega_{i}^{2}\right) \frac{\partial H}{\partial I_{i}} M\left|\omega_{i}\right| Q_{i}
\end{gathered}
$$

and can be integrated exactly to give

$$
\begin{aligned}
& Q_{1}(\alpha, t)=a_{1}(\alpha) \mathrm{e}^{\Omega_{1}(\alpha) t}+b_{1}(\alpha) \mathrm{e}^{-\Omega_{1}(\alpha) t} \\
& Q_{2}(\alpha, t)=a_{2}(\alpha) \mathrm{e}^{\Omega_{2}(\alpha) t}+b_{2}(\alpha) \mathrm{e}^{-\Omega_{2}(\alpha) t}
\end{aligned}
$$


where we have introduced the effective frequencies $\Omega_{i}=$ $\partial \mathscr{H} / \partial I_{i}$. The expressions for the momenta $\mathbf{P}$ are directly obtained from eqn. (24) and have a similar form. The canonical transformation maps the initial conditions in eqn. (19) into the new phase space determining $\left[\mathbf{Q}_{0}(\alpha), \mathbf{P}_{0}(\alpha)\right]$, and hence $a_{i}(\alpha), b_{i}(\alpha), \Omega_{i}(\alpha)$, and $I_{i}(\alpha)$, which are known polynomial functions of $\mathbf{Q}_{0}$ and $\mathbf{P}_{0}$. It is also convenient to rewrite $W(\alpha, t)$ directly in terms of the new canonical variables. The phase integral along any contour in the phase space can then be expressed as

$$
\int \mathbf{p} \mathrm{d} \mathbf{q}=\int \mathbf{P} \mathrm{d} \mathbf{Q}+S(\mathbf{P}, \mathbf{q})-\mathbf{P} \frac{\partial S}{\partial \mathbf{P}}
$$

Using eqn. (27) in eqn. (2) and (3), one then gets

$$
\begin{aligned}
W(t, \alpha)= & \tilde{W}_{0}(\alpha)+\int_{\alpha, 0}^{\alpha, t} \mathbf{P}(\alpha, \tau) \mathrm{d} \mathbf{Q}(\alpha, \tau) \\
& +S(\mathbf{P}, \mathbf{q})-\mathbf{P} \frac{\partial S}{\partial \mathbf{P}}
\end{aligned}
$$

with

$$
\tilde{W}_{0}(\alpha)=\int^{\alpha} \mathbf{P}_{0}\left(\alpha^{\prime}\right) \mathrm{d} \mathbf{Q}_{0}\left(\alpha^{\prime}\right)
$$

Thus, apart from two last terms in eqn. (28), $W$ preserves its form in the new variables. Moreover the integral in eqn. (28) is taken at a fixed value of the parameter $\alpha$ and can be rewritten as

$$
\int_{\alpha, 0}^{\alpha, t} \mathbf{P} \mathrm{d} \mathbf{Q}=\sum_{i=1,2} \int_{Q_{i}(\alpha, 0)}^{Q_{i}(\alpha, t)} \sqrt{I_{i}\left|\omega_{i}\right|-\frac{M \omega_{i}^{2} Q_{i}^{2}}{2}} \mathrm{~d} Q_{i}
$$

which is the same as a harmonic potential barrier. Note also that, except for the term $\operatorname{Re}(\mathbf{n v}) /|(\mathbf{n v})|$, the form of eqn. (16) is invariant in any canonical variables. We assume that the surface $S_{f}$ can be chosen in such a way that the flux does not vary significantly with its location. It means that the main contribution to the integral in eqn. (16) comes from the area where the $\operatorname{Im} \nabla W$ is small. Hence the factor $\operatorname{Re}(\mathbf{n v}) /|(\mathbf{n v})| \simeq 1$ and one may also neglect the two last terms in eqn. (28) since they are just functions of the canonical variables (if the latter are real then we can skip such terms). This reduces the problem of multidimensional tunnelling to the consideration of the harmonic case while the only remaining trace of the initial anharmonicity is the difference between $\omega_{i}$ and the effective frequencies $\Omega_{i}$ in eqn. (26). Thus, once specified, $\mathbf{Q}_{0}(\alpha)$, $\mathbf{P}_{0}(\alpha)$ in the new canonical variables, eqn. (26) and (28) together with the appropriate expressions for $\mathbf{P}(\alpha, t)$ give the complex-valued $W$ in the algebraic form and provide the full information about tunnelling. However, even after all of the above simplifications, such a numerical analysis is not easy and the crucial obstacle stems from the initial conditions $\mathbf{Q}_{0}$ and $P_{0}$. Although these functions can be accurately calculated for real $\alpha$, their extrapolation in the complex $\alpha$ space is not a trivial task. In this paper, we use the above similarity with the harmonic case to formulate a strategy for the hopping method. This is very simple in practice, and allows one to estimate the tunnelling probability with exponential accuracy.

\subsection{The hopping method}

As shown above, the canonical perturbation theory allows one to reduce the anharmonicities in the initial Hamiltonian, and to rewrite the exponential factor $W$ for the tunnelling probability in a form equivalent to the parabolic barrier case. Of course, we assume that the perturbation treatment is performed with the required accuracy, which can always be satisfied in practice. Moreover, under additional conjectures the expression for the tunnelling probability does not depend on the choice of the canonical variables. This gives a hint on how to implement the hopping method by using "actions" $I_{i}$ to calculate the hopping probability in a way similar to the harmonic case. According to the hopping recipe, we consider a bunch of $N$ trajectories which describe the scattering process and calculate for every trajectory the values $I_{i}$. For classically nonreactive trajectories $I_{2}<0$, and the hopping probability $P_{n}$ is determined as $P_{n}=\exp \left[\left(2 \pi I_{2 n}\right) / \hbar\right]$ where $n=1, \ldots N$ labels the different trajectories in the bunch. Then the tunnelling probability $P_{\text {tun }}$ is given by

$$
P_{\text {tun }}=\frac{1}{N} \sum_{n=1}^{N} P_{n}
$$

It is worthwhile to stress that the limitation of such an approach is related to the initial conditions in eqn. (19), and it has nothing to do with the anharmonicity of the potential. Thus, if one could continue the family of trajectories into the tunnelling region with complex time and real $\alpha$, the calculation of $\operatorname{Im} W$ would give exactly $\pi\left|I_{2}\right|$. In fact, by using eqn. (17), the probability in eqn. (16) becomes just the average value of the exponential factor $\exp \left[\left(2 \pi I_{2 n}\right) / \hbar\right]$ over the bunch of the trajectories, i.e., assumes the form of eqn. (31). As we have pointed out above such an intuitive picture of multidimensional tunnelling relies on our experience with the 1D problem. ${ }^{27}$ Generally the integration in eqn. (16) is performed along a contour in complex $\alpha$-space, and one must take into account the $\alpha$-dependence of $I_{i}$ at complex-valued $\alpha$. In the simplest "separable" case when one can neglect this dependence and deem the "actions" $I_{i}$ to be constant within the whole family, the calculation of Im $W$ according to eqn. (30) then gives again $\pi\left|I_{2}\right|$. Thus, the exponential factor in eqn. (16) is a constant, giving the tunnelling probability with an exponential accuracy. Of course this result is also reproduced by the hopping method since $I_{2 n}$ and $P_{n}$ in eqn. (31) are the same for all $n$. The hopping recipe looks realistic also in the more general case. Thus, if the function $-I_{2}(\alpha)$ has a minimum at $\alpha=\alpha_{0}$, one can approximately treat $J_{i}$ as constants in the vicinity of $\alpha_{0}$; note that the energy is the same for all the trajectories in the bunch and therefore $I_{1}$ has also an extrema at $\alpha_{0}$. This is close in spirit to the concept of "locally conserved actions" in transition state theory although in $\alpha$ space rather than in time. The value of $I_{2}\left(\alpha_{0}\right)$ determines then the tunnelling probability with an exponential accuracy which is also consistent with the hopping recipe in eqn. (31) where the main contribution comes from the trajectories with $I_{2 n}$ close to $I_{2}\left(\alpha_{0}\right)$. Such a situation is realized in pure tunnelling if $\alpha_{0}$ corresponds to the trajectory having the classical turning point $\mathbf{p}=0$. The disregard of $\alpha$ dependence in $I_{i}$ can be recast as changes in the initial conditions which do not affect the position of the turning point but modify the form of the caustics. The numerical evaluation of the prefactor requires an accurate approximation of $\mathbf{Q}_{0}(\alpha)$ and $\mathbf{P}_{0}(\alpha)$ in the vicinity of $\alpha_{0}$ and a study of eqn. (26). We refrain from such a detailed analysis here, and postpone this and related issues to a forthcoming publication.

\section{Numerical illustration of the hopping method: $\mathrm{H}+\mathbf{H}_{\mathbf{2}}$ collinear exchange reaction}

To provide a numerical test of the proposed hopping method we consider the collinear $\mathrm{H}+\mathrm{H}_{2}$ exchange reaction at collision energies near the classical threshold. All calculations have been done using the popular $\mathrm{H}_{3}$ DMBE potential energy surface $:^{33}$ this has a saddle point which is characterized by a classical barrier height for reaction of $9.65 \mathrm{kcal} \mathrm{mol}^{-1}$ at a geometry of $D_{\infty \mathrm{h}}$ symmetry with a characteristic bond length of $R_{\mathrm{H}^{\prime}-\mathrm{H}}=1.755 a_{0}$. For comparison purposes, we have also carried out the exact solution of the $2 \mathrm{D}$ quantum problem using the same potential energy surface. For this we have 
implemented the $R$-matrix propagation technique ${ }^{39}$ in hyperspherical coordinates defined as usually carried out for collinear three-atom systems. ${ }^{40}$ The whole interval in the hyperradius $\rho \in\left[\rho_{i}, \rho_{f}\right]$ has then been divided into a number of sectors, and within each sector the $R$-matrix basis was calculated using the smooth variable discretization (SVD) technique. ${ }^{41}$ Zero boundary conditions at $\rho_{i}$ for the $R$-matrix have been guaranteed by the choice of the Jacobi polynomials $P_{n}^{(0,2)}$ to construct the discrete variable representation (DVR) quadrature for the first sector. After propagating the $R$-matrix up to $\rho_{f}$ the asymptotic solutions in the Jacobi coordinates were used to calculate $K$ - and $S$-matrices in the usual way. Most calculations are rather well known and we omit a complete description of the numerical procedure. The details of the implemented SVD approach for the collisional problem and the matching procedure can be found in ref. 42 .

In the scope of the hopping method we first have to calculate the anharmonic force constants, i.e., the partial derivatives of the potential function $V$ with respect to the normal coordinates at the transition state. We use the notation

$$
F_{i j}^{(2)}=\frac{\partial^{2} V}{\partial q_{i} \partial q_{j}}, \quad F_{i j k}^{(3)}=\frac{\partial^{3} V}{\partial q_{i} \partial q_{j} \partial q_{k}}, \text { etc. }
$$

Thus the upper indexes indicate the order of anharmonicity, and the normal coordinates $q_{i}$ for $\mathrm{H}_{3}$ are determined at the transition state $\left(R_{\mathrm{H}-\mathrm{H}}=1.7546353 a_{0}\right)$ as

$$
\begin{array}{lll}
\Delta X_{1}^{1}=-\sqrt{\frac{1}{6}} q_{4} & \Delta X_{1}^{2}=\sqrt{\frac{2}{3}} q_{4} & \Delta X_{1}^{3}=-\sqrt{\frac{1}{6}} q_{4} \\
\Delta X_{2}^{1}=-\sqrt{\frac{1}{6}} q_{3} & \Delta X_{2}^{2}=\sqrt{\frac{2}{3}} q_{3} & \Delta X_{2}^{3}=-\sqrt{\frac{1}{6}} q_{3} \\
\Delta X_{3}^{1}=\frac{q_{1}}{\sqrt{2}}-\sqrt{\frac{1}{6}} q_{2} & \Delta X_{3}^{2}=\sqrt{\frac{2}{3}} q_{2} & \\
\Delta X_{3}^{3}=-\frac{q_{1}}{\sqrt{2}}-\sqrt{\frac{1}{6}} q_{2} & &
\end{array}
$$

where $\Delta X_{i}^{k}$ are displacement along the $i$-th direction for the nucleus $k$. To calculate the force constants it is enough to consider only one of the degenerate bending modes $q_{3}$ or $q_{4}$. To evaluate the partial derivatives we introduce the auxiliary functions

$$
\Phi^{j}(z)=V\left(S_{1}^{j} z, S_{2}^{j} z, S_{3}^{j} z\right)
$$

where $V(\mathbf{q})$ in the potential energy surface written in terms of the normal coordinates $q_{i}$, and $S_{i}^{j}(i=1,2,3)$ are some arbitrary sets of stretches. Using a NAG LIBRARY subroutine we then calculate the derivatives $\mathrm{d}^{k} \Phi^{j} / \mathrm{d} z^{k}$, which are linear combinations of the partial derivatives $F^{(k)}$. Thus, by calculating the derivatives of different $\Phi^{j}$ we have a system of linear equations to determine all the force constants $F^{(k)}$. The calculated values are given in Table 1.

For the collinear version of the reaction only the symmetric stretching and antisymmetric tunnelling modes $\left(q_{1}, q_{2}\right)$ are relevant, while the anharmonic approximation in the vicinity of

Table 1 Harmonic and anharmonic force constants $F^{(n)}$ in normal coordinates for the $\mathrm{H}_{3}$ DMBE potential energy surface at the transition state $\left(R_{\mathrm{H}-\mathrm{H}}=1.7546353 \quad a_{0}\right): 1=$ symmetric stretching, $2=$ tunnelling mode, $3=$ degenerate bending modes

\begin{tabular}{llllll}
\hline$i j$ & $F_{i j}^{(2)}$ & $i j k$ & $F_{i j k}^{(3)}$ & $i j k l$ & $F_{i j k l}^{(4)}$ \\
\hline 11 & 0.1629385 & 111 & -0.33812 & 1111 & 0.614237 \\
22 & -0.0850237 & 222 & 0.0 & 2222 & 11.8587 \\
33 & 0.03086142 & 112 & 0.0 & 3333 & 0.59467 \\
& & 122 & -0.79698 & 1122 & 1.87 \\
& & 133 & 0.15717 & 1133 & -0.519 \\
& & 233 & 0.0 & 2233 & -0.937
\end{tabular}

All values are given in atomic units $\left(E_{\mathrm{h}} / a_{0}^{n}\right)$ the saddle point assumes the form

$$
\begin{aligned}
V\left(q_{1}, q_{2}\right)= & \frac{F_{11}}{2} q_{1}^{2}+\frac{F_{22}}{2} q_{2}^{2}+\frac{F_{111}^{(3)}}{6} q_{1}^{3}+\frac{F_{122}^{(3)}}{2} q_{1} q_{2}^{2} \\
& +\frac{F_{1111}^{(4)}}{24} q_{1}^{4}+\frac{F_{2222}^{(4)}}{24} q_{2}^{4}+\frac{F_{1122}^{(4)}}{4} q_{1}^{2} q_{2}^{2}
\end{aligned}
$$

where odd terms in $q_{2}$ are missing owing to symmetry reasons.

The numerical procedure to calculate the reaction probability can now be summarized as follows. We start a bunch of $N$ classical trajectories which describe in the asymptotic region the incoming wave function for a given collision energy, and assume a homogeneous distribution of the phase for the diatomic $\mathrm{H}_{2}$ in the ground vibrational state. When the trajectories move in the vicinity of the saddle point of the potential energy surface, we expect the anharmonic expansion in eqn. (33) to be appropriate and use the canonical perturbation approach to calculate the two classical integrals of motion $I_{1}$ and $I_{2}$. Since we consider the Taylor expansion of the potential energy surface only with an accuracy up to quartic terms, two successive canonical transformations are sufficient to accomplish our goal. The first of them removes the third order anharmonicity from the Hamiltonian, while the second converts the terms of the fourth order into a quadratic form of $I_{i}$ in eqn. (23). The explicit form of the appropriate generators $S^{(3)}$ and $S^{(4)}$ are given in the Appendix. For every trajectory we prescribe a reaction probability which is equal to unity if the trajectory is reactive in the classical sense, and $\exp \left[\left(2 \pi\left|I_{2}\right|\right) / \hbar\right]$ otherwise. The main numerical problem arises from the fact that eqn. (33) is applicable only in a relatively small area of the coordinate space. Fig. 1 presents a comparison between the DMBE potential energy surface ${ }^{33}$ and its approximation given by eqn. (33). An illustrative example of classical trajectories used in the scattering calculations is also given on the same plot. To provide the numerical test of the applicability of the perturbative approach, we have first performed the calculations of the actions $I_{i}$ along the trajectories moving on the model potential of eqn. (33) rather than in the real one. Fig. 2 shows the time-behaviour of the action $I_{2}$ along such a trajectory with an energy of about $0.27 \mathrm{eV}$. From here onwards we refer the total energy to the ground state of $\mathrm{H}_{2}$. Clearly visible from Fig. 2 is a plateau where $I_{2}$ remains nearly constant during almost 200 atomic units of time. Thus, for this trajectory the two-step canonical transformation provides a reliable approximation for the classical integrals of motion $I_{i}$ and the trajectory moves for a long time in the region of applicability of the perturbative approach. The width of the plateau decreases with energy and the test provides a numerical estimation for the energy region

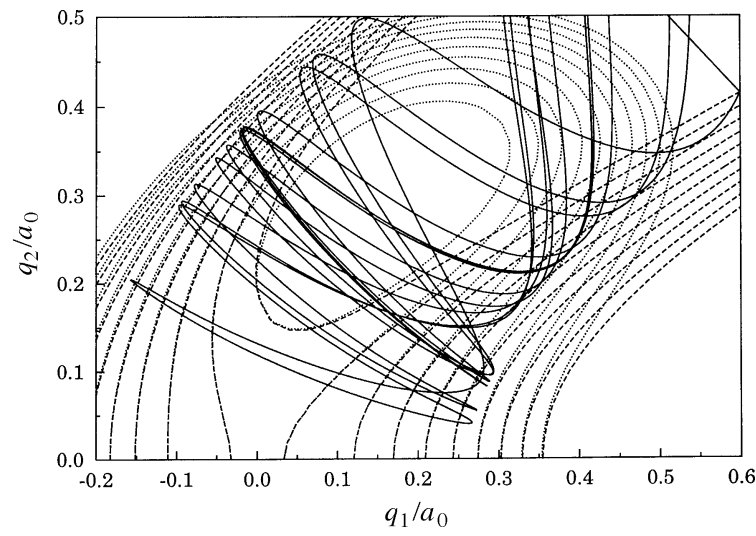

Fig. 1 DMBE potential energy surface in normal coordinates and its anharmonic expansion in the vicinity of the saddle point. Classical trajectories correspond to the collisional energy $E_{\mathrm{tr}} \approx 0.27 \mathrm{eV}$. 


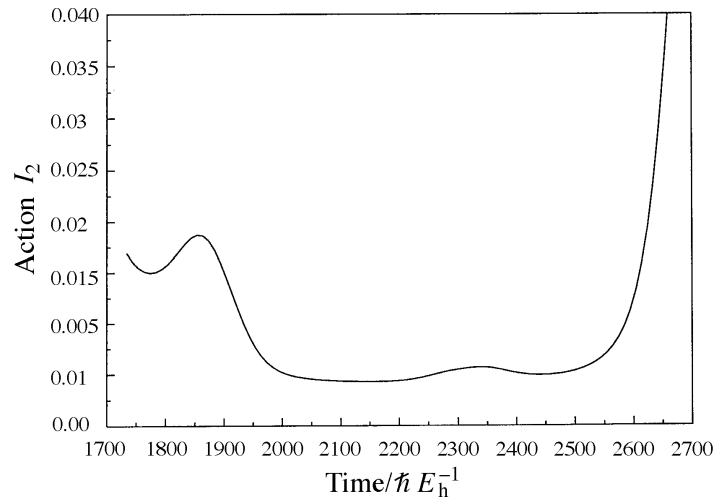

Fig. 2 Time behavior of the "action" $I_{2}$ calculated along the classical trajectory in the model potential of eqn. (33).

where the two-step perturbative treatment is sufficient. The anharmonicity in the considered case is rather strong, and for $E<0.26 \mathrm{eV}$ we cannot see any apparent plateau such as that in Fig. 2. For trajectories on the real potential energy surface the evaluation of $I_{i}$ is less clear. We cannot expect a timeindependent behavior even at sufficiently high energies since the trajectories spend only a short time in the region where the expansion in eqn. (33) is valid. Hence it is not quite evident at what moment the actions should be calculated. In this work the following procedure has been employed. For every trajectory in the bunch we have stored its coordinates and momenta at the point where the difference between the

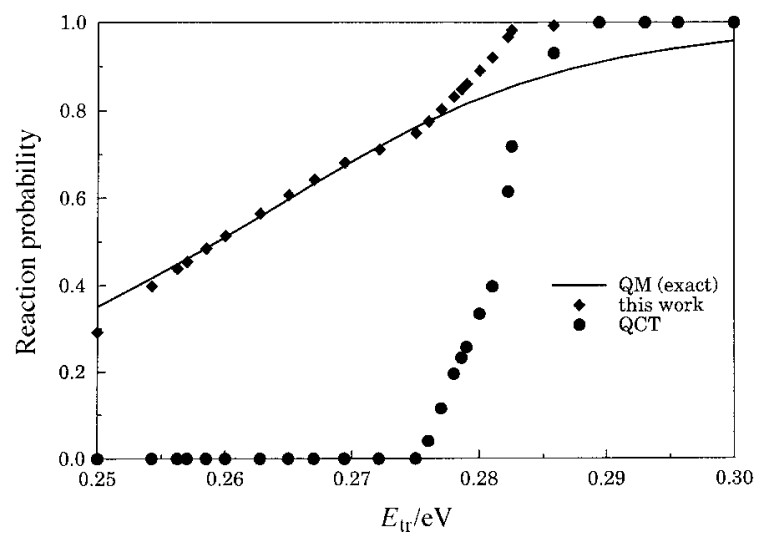

Fig. 3 Exchange probability for the title reaction at collisional energies near the classical threshold: exact quantum (-); hopping method $(\diamond)$. The purely classical reactivity $(\mathbf{O})$ is also depicted.

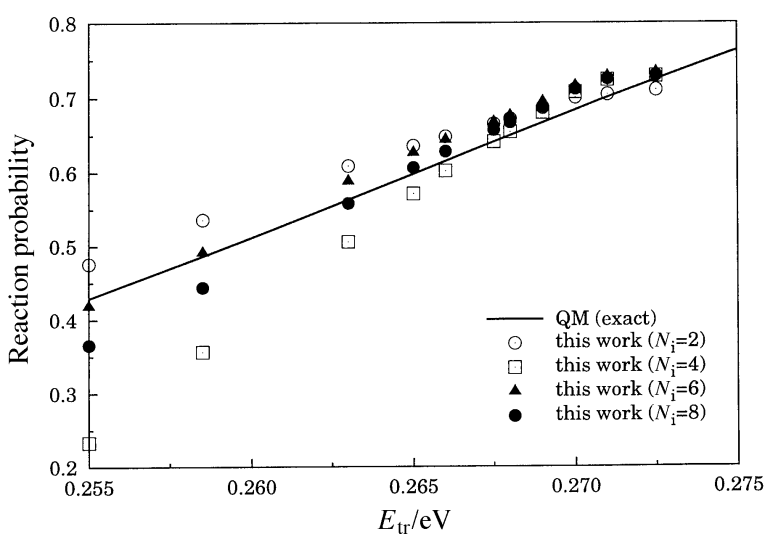

Fig. 4 Exchange probability for the title reaction. The hopping probability is calculated using eqn. (34) with the number of iterations $N_{\mathrm{i}}=2,4,6,8$.
DMBE potential energy surface and eqn. (33) becomes minimal and then used these values to calculate the new coordinates $(\mathbf{Q}, \mathbf{P})$ and the hopping probability.

The results of the calculations are presented on Fig. 3. The solid line shows the exact quantum probability $\left(S_{00}\right.$ element of the $S$-matrix) and we also plot, for comparison, the classical probability without tunnelling correction (QCT). One can see the excellent agreement between the exact and semiclassical results at collisional energies below the classical threshold $\left(E_{\mathrm{tr}} \simeq 0.275 \mathrm{eV}\right)$. We cannot expect such an agreement at high energies near the classical threshold where quantum interference plays an important role. At the same time for the energies below $0.25 \mathrm{eV}$ the classical trajectories pass too far away from the saddle point and the approximation in eqn. (33) becomes inappropriate. For this reason the calculated semiclassical probability shows some chaotic jumps at smaller $E_{\text {tr }}$ which we have omitted to display in the plot of Fig. 1. As mentioned above, even without paying attention to the accuracy of eqn. (33), the two-step perturbative approach is probably not enough for energies below $0.26 \mathrm{eV}$ and the good agreement at these energies is rather surprising. A rough test to determine the possibility of dismissing the higher anharmonicity terms in the Hamiltonian can be done by determining iteratively the new momenta in eqn. (22) as

$$
\mathbf{P}_{\mathrm{i}+1}=\mathbf{p}-\frac{\partial S\left(\mathbf{P}_{\mathrm{i}}, \mathbf{q}\right)}{\partial \mathbf{q}}
$$

Note that eqn. (21) does not need to be considered since it directly gives the new coordinates $\mathbf{Q}$ in terms of $(\mathbf{P}, \mathbf{q})$. Thus the nontrivial part of the generators $S^{(3)}, S^{(4)}$ are proportional to the anharmonic force constants $F^{(3)} ; F^{(3) 2}, F^{(4)}$ and the truncation of the perturbation expansion of the Hamiltonian implies that it is possible to omit higher terms. Generally the iterative procedure in eqn. (34) is not reliable for solving eqn. (22), and its poor convergence indicates that the higher degrees of the force constants are very important to calculate the integrals of motion. Fig. 4 shows the reaction probability using eqn. (34) with the iteration number $N_{\mathrm{i}}=2,4,6,8$. Once again, we conclude that the truncated perturbative procedure is mostly reliable above $\sim 0.26 \mathrm{eV}$. This is the threshold area where tunnelling plays a crucial role while the classical reactive probability is zero. Thus, near the threshold, the proposed hopping method provides a simple tool to incorporate tunnelling effects in classical trajectory simulations.

\section{Concluding remarks}

In this work we have considered the problem of multidimensional tunnelling in reactive scattering dynamics. By neglecting interference between different branches of the semiclassical solution, we have derived a simple expression for the tunnelling probability and shown that the method of classical canonial transformations reduces the problem to the harmonic barrier case. This analogy has then been used to suggest a simple hopping method which allows the incorporation of tunnelling effects in classical trajectories simulations.

To calculate the classical integrals of motion $I_{i}$ and hopping probability, we have employed the Gustavson-Birkhoff ${ }^{30-32}$ canonical perturbation theory in the vicinity of the transition state. We have tested the proposed numerical recipe for the collinear $\mathrm{H}+\mathrm{H}_{2}$ exchange reaction and found an excellent agreement with the exact quantum results at collisional energies near the classical threshold. In the general case, when the exact solution is unaffordable, the examination of accuracy of the hopping method requires an analysis of a system of algebraic equations which provides the complex-valued solution of the Hamilton-Jacobi equation in the tunnelling region. This depends on the distribution of the values $I_{i}$ among the trajectories, and requires an analytical approximation for the 
initial conditions $\left(\mathbf{P}_{\mathbf{0}}, \mathbf{Q}_{\mathbf{0}}\right)$ in the transformed canonical variables. Work along these lines is in progress in our group.

\section{Acknowledgements}

This work has been supported by the Fundação para a Ciência e Tecnologia, Portugal, under programmes PRAXIS XXI and FEDER. G.V.M. thanks the Institute of Structural Macrokinetics, Chernogolovka, Moscow Region, Russia, for leave of absence.

\section{Appendix Canonical transformation at the transition state of the $\mathrm{H}_{3}$ system}

The details of the implemented perturbative approach can be found in ref. 32 and we simply outline here the main steps. In the vicinity of the saddle point of the potential energy surface we use the Taylor series expansion up to fourth order terms and write the Hamiltonian in the form

$$
H(\mathbf{q}, \mathbf{p})=\sum_{i=1} H_{0 i}+V^{(3)}(\mathbf{q})+V^{(4)}(\mathbf{q})
$$

where $H_{0 i}=\left(p_{i}^{2} / 2 \mu\right)+\left(\mu \omega_{i}^{2} / 2\right) q_{i}^{2}$, and the anharmonic terms are meant to be proportional to the force constants $F^{(3)}$ and $F^{(4)}$.

After canonical transformation with the second-type generator in eqn. (20), the Hamiltonian in the new variables $(\mathbf{Q}, \mathbf{P})$ becomes

$$
\begin{aligned}
H(\mathbf{P}, \mathbf{Q})= & H+\{S, H\}+\frac{1}{2}\{S,\{S, H\}\} \\
& +\frac{1}{2} \sum_{i=1}\left(S_{Q_{i}}\left\{H, S_{P i}\right\}+S_{P_{i}}\left\{H, S_{Q_{i}}\right\}\right)+\cdots
\end{aligned}
$$

where $\{$,$\} denotes a Poisson bracket, S$ differs from $S(\mathbf{P}, \mathbf{q})$ only in the fact that the new coordinates $\mathbf{Q}$ replace $\mathbf{q}$, and we have used the compact notation $S_{Q_{i}} \equiv \partial S / \partial Q_{i}$ and $S_{P_{i}} \equiv$ $\partial S / \partial P_{i}$. The first canonical transformation is chosen to remove the cubic terms $\sim F^{(3)}$ from the Hamiltonian and the appropriate generator $S^{(3)}$ must satisfy the equation

$$
V^{(3)}=\left\{H_{0}, S^{(3)}\right\}
$$

The solution of eqn. (A3) is formally written as

$$
S^{(3)}=\mathbf{D}^{-1} V^{(3)}
$$

where the operator $D$ is

$$
\mathbf{D}=\left\{H_{0},\right\}=\sum_{i}\left(\mu_{i} \omega_{i}^{2} Q_{i} \frac{\partial}{\partial P_{i}}-\mu_{i}^{-1} P_{i} \frac{\partial}{\partial Q_{i}}\right)
$$

A convenient way to calculate the right-hand side in eqn. (A4) is to introduce the new coordinates $\left(z_{i}, z_{i}^{*}\right)^{32}$

$$
\begin{aligned}
P_{k} & =\sqrt{\frac{\mu}{2}} i\left(z_{k}^{*}-z_{k}\right) \\
Q_{k} & =\frac{1}{\omega_{k}} \sqrt{\frac{1}{2 \mu}}\left(z_{k}^{*}+z_{k}\right)
\end{aligned}
$$

In terms of $\left(z_{i}, z_{i}^{*}\right), \mathbf{D}$ assumes the form

$$
\mathbf{D}=\sum_{k=1}^{N} i \omega_{k}\left(z_{k} \frac{\partial}{\partial z_{k}}-z_{k}^{*} \frac{\partial}{\partial z_{k}^{*}}\right)
$$

while the harmonic Hamiltonians become

$$
H_{0 i}=z_{i}^{*} z_{i}
$$

Any function of canonical variables can be written now as a sum of monomials $\Pi_{i} z_{i}^{l_{i}} z_{i}^{* m_{i}}$. These are eigenfunctions of $\mathbf{D}$ with eigenvalues $\Sigma_{i} i \omega_{i}\left(l_{i}-m_{i}\right)$ and hence are also the eigen- functions of $\mathbf{D}^{-1} \cdot V^{(3)}$ does not contain terms with zero eigenvalues, and the calculation of eqn. (A4) is straightforward

$$
\begin{aligned}
S(\mathbf{P}, \mathbf{Q})= & \frac{F_{111}^{(3)}}{3}\left(\frac{P_{1}^{3}}{3 \omega_{1}^{3} M^{3}}+\frac{P_{1} Q_{1}^{2}}{2 \omega_{1}^{2} M}\right) \\
& +\frac{F_{122}^{(3)}}{2}\left(\frac{2 \omega_{2}^{2}-\omega_{1}^{2}}{\omega_{1}^{2}\left(4 \omega_{2}^{2}-\omega_{1}^{2}\right)} \frac{P_{1} Q_{2}^{2}}{M}\right. \\
& \left.+\frac{2 P_{2} Q_{1} Q_{2}}{\left(4 \omega_{2}^{2}-\omega_{1}^{2}\right) M}+\frac{2 P_{1} P_{2}^{2}}{\omega_{1}^{2}\left(4 \omega_{2}^{2}-\omega_{1}^{2}\right) M^{3}}\right)
\end{aligned}
$$

The quartic anharmonicity in the transformed Hamiltonian in eqn. (A2) is handled in a similar way. The only difference emanates from the presence of the quartic tems $\left(z_{1} z_{1}^{*}\right)^{l_{1}}\left(z_{2} z_{2}^{*}\right)^{l_{2}}$ referred to as "null space terms". For these terms the operator $\mathbf{D}^{-1}$ is not defined and they must be omitted in calculating $S^{(4)}$, which assumes the form

$$
\begin{aligned}
& S(\mathbf{P}, \mathbf{Q})=\left(\frac{17 F_{111}^{(3) 2}}{576 \omega_{1}^{6} M^{4}}+\frac{F_{1111}^{(4)}}{64 \omega_{1}^{4} M^{3}}\right) P_{1}^{3} Q_{1} \\
& +\left(\frac{7 F_{111}^{(3) 2}}{576 \omega_{1}^{4} M^{2}}+\frac{5 F_{1111}^{(4)}}{192 \omega_{1}^{2} M}\right) P_{1} Q_{1}^{3} \\
& +\left(\frac{\left(20 \omega_{2}^{2}-3 \omega_{1}^{2}\right) F_{122}^{(3) 2}}{64 \omega_{2}^{4}\left(4 \omega_{2}^{2}-\omega_{1}^{2}\right)^{2} M^{4}}+\frac{F_{2222}^{(4)}}{64 \omega_{2}^{4} M^{3}}\right) P_{2}^{3} Q_{2} \\
& +\left(\frac{5 F_{2222}^{(4)}}{192 \omega_{2}^{2} M}+\frac{\left(12 \omega_{2}^{2}-5 \omega_{1}^{2}\right) F_{122}^{(3) 2}}{64 \omega_{2}^{2}\left(4 \omega_{2}^{2}-\omega_{1}^{2}\right)^{2} M^{2}}\right) P_{2} Q_{2}^{3} \\
& +\left(\frac{\left(16 \omega_{2}^{6}-16 \omega_{2}^{4} \omega_{1}^{2}+10 \omega_{2}^{2} \omega_{1}^{4}-\omega_{1}^{6}\right) F_{122}^{(3) 2}}{8 \omega_{2}^{2} \omega_{1}^{4}\left(\omega_{2}^{2}-\omega_{1}^{2}\right)\left(4 \omega_{2}^{2}-\omega_{1}^{2}\right)^{2} M^{4}}\right. \\
& -\frac{F_{111}^{(3)} F_{122}^{(3)}}{16 \omega_{2}^{2}\left(4 \omega_{2}^{2}-\omega_{1}^{2}\right)\left(\omega_{2}^{2}-\omega_{1}^{2}\right) M^{4}} \\
& \left.+\frac{F_{1122}^{(4)}}{16 \omega_{2}^{2}\left(\omega_{1}^{2}-\omega_{2}^{2}\right) M^{3}}\right) P_{1}^{2} P_{2} Q_{2} \\
& +\left(\frac{\left(5 \omega_{1}^{2}+4 \omega_{2}^{2}\right) F_{122}^{(3) 2}}{8 \omega_{1}^{2}\left(\omega_{1}^{2}-\omega_{2}^{2}\right)\left(4 \omega_{2}^{2} \omega_{1}^{2}\right)^{2} M^{4}}\right. \\
& +\frac{F_{111}^{(3)} F_{122}^{(3)}}{48 \omega_{1}^{4}\left(\omega_{2}^{2}-\omega_{2}^{2}\right) M^{4}} \\
& \left.+\frac{F_{1122}^{(4)}}{16 \omega_{1}^{2}\left(\omega_{2}^{2}-\omega_{1}^{2}\right) M^{3}}\right) P_{2}^{2} P_{1} Q_{1} \\
& +\left(\frac{\left(12 \omega_{2}^{4}-4 \omega_{1}^{2} \omega_{2}^{2}+\omega_{1}^{4}\right) F_{122}^{(3) 2}}{8 \omega_{2}^{2}\left(\omega_{1}^{2}-\omega_{2}^{2}\right)\left(4 \omega_{2}^{2}-\omega_{1}^{2}\right)^{2} M^{2}}\right. \\
& +\frac{\left(2 \omega_{2}^{2}-\omega_{1}^{2}\right) F_{111}^{(3)} F_{122}^{(3)}}{16 \omega_{2}^{2}\left(4 \omega_{2}^{2}-\omega_{1}^{2}\right)\left(\omega_{2}^{2}-\omega_{1}^{2}\right) M^{2}} \\
& \left.+\frac{\left(\omega_{1}^{2}-\omega_{2}^{2}\right) F_{1122}^{(4)}}{16 \omega_{2}^{2}\left(\omega_{1}^{2}-\omega_{2}^{2}\right) M}\right) P_{2} Q_{1}^{2} Q_{2} \\
& +\left(\frac{\left(12 \omega_{2}^{4}-5 \omega_{1}^{2} \omega_{2}^{2}+2 \omega_{1}^{4}\right) F_{122}^{(3) 2}}{8 \omega_{1}^{2}\left(\omega_{2}^{2}-\omega_{1}^{2}\right)\left(4 \omega_{2}^{2}-\omega_{1}^{2}\right)^{2} M^{2}}\right. \\
& +\frac{\left(4 \omega_{2}^{4}+2 \omega_{1}^{4}-9 \omega_{1}^{2} \omega_{2}^{2}\right) F_{111}^{(3)} F_{122}^{(3)}}{48 \omega_{1}^{4}\left(4 \omega_{2}^{2}-\omega_{1}^{2}\right)\left(\omega_{2}^{2}-\omega_{1}^{2}\right) M^{2}} \\
& \left.+\frac{\left(\omega_{2}^{2}-2 \omega_{1}^{2}\right) F_{1122}^{(4)}}{16 \omega_{1}^{2}\left(\omega_{2}^{2}-\omega_{1}^{2}\right) M}\right) P_{1} Q_{2}^{2} Q_{1}
\end{aligned}
$$

After the second canonical transformation the Hamiltonian still contains the "null space terms" of fourth order $\sim F^{(3) 2}$, $F^{(4)}$, which are simply the products of harmonic Hamiltonians $H_{0 \mathrm{i}}$ as is seen from eqn. (A9).

\section{References}

1 S. Coleman and F. de Luccia, Phys. Rev. D., 1980, 21, 3305

2 J. S. Langer, Ann. Phys., 1967, 41, 108.

3 C. G. Gallen and S. Coleman, Phys. Rev. D., 1977, 16, 1762. 
4 S. Coleman, Phys. Rev. D., 1977, 15, 2929.

5 T. Banks, C. Bender and T. Wu, Phys. Rev. D., 1973, 8, 3366.

6 H. M. Van Horn and E. E. Salpeter, Phys. Rev., 1967, 157, 751.

7 A. O. Caldeira and A. J. Leggett, Ann. Phys., 1983, 149, 374.

8 V. A. Benderskii, D. E. Makarov and C. A. Wight, Adv. Chem. Phys., 1994, 88, 1

9 N. Makri and W. H. Miller, J. Chem. Phys., 1987, 86, 1451.

10 N. Makri and W. H. Miller, J. Chem. Phys., 1987, 87, 5781.

11 S. Takada and H. Nakamura, J. Chem. Phys., 1994, 100, 98.

12 P. Bowcock and R. Gregory, Phys. Rev. D., 1991, 44, 1774.

13 Z. H. Huang, T. E. Feuchtwang, P. H. Culter and E. Kazes, Phys. Rev. A, 1990, 41, 32.

14 R. Rajaraman, Phys. Rev., 1975, 21, 227

15 J. F. Willemsen, Phys. Rev. D., 1979, 20, 3292

16 A. Auerbach and S. Kivelson, Nucl. Phys. B, 1985, 257, 799.

17 W. H. Miller, Adv. Chem. Phys., 1974, 25, 69.

18 W. H. Miller, Adv. Chem. Phys., 1975, 30, 74.

19 A. Schmid, Ann. Phys., 1986, 170, 333.

20 V. A. Benderskii, S. Yu. Grebenshchikov and G. V. Mil'nikov, Chem. Phys., 1995, 194, 1.

21 T. F. George and W. H. Miller, J. Chem. Phys., 1972, 56, 5668.

22 T. F. George and W. H. Miller, J. Chem. Phys., 1972, 57, 2458

23 N. Makri and W. H. Miller, J. Chem. Phys., 1989, 91, 4026.

24 S. Keshavamurthy and W. H. Miller, Chem. Phys. Lett., 1993, $205,96$.

25 J. C. Tully and R. K. Preston, J. Chem. Phys., 1971, 55, 562.

26 D. W. McLaughlin, J. Math. Phys., 1972, 13, 1099.
27 A. J. C. Varandas and G. V. Mil'nikov, Chem. Phys. Lett., 1996, 259, 605 .

28 M. Alonso and E. J. Finn, Fundamental University Physics, Addison-Wesley, Reading, MA, 1967, vol. II, p. 774.

29 S. Takada, J. Chem. Phys., 1996, 104, 3742.

30 G. D. Birkhoff, Dynamical Systems, American Mathematical Society, New York, 1966.

31 F. G. Gustavson, Astrophys. J., 1966, 71, 670.

32 R. T. Swimm and J. B. Delos, J. Chem. Phys., 1979, 71, 1706.

33 A. J. C. Varandas, F. B. Brown, C. A. Mead, D. G. Truhlar and N. C. Blais, J. Chem. Phys., 1987, 86, 6258.

34 V. P. Maslov and M. V. Fedoriuk, Semiclassical Approach in Quantum Mechanics, Reidel, Dordrecht, 1981.

35 J. B. Delos, Adv. Chem. Phys., 1986, LXV, 161.

36 M. Wilkinson, Physica, 1986, 21, 341.

37 N. Bleistein, Mathematical Methods for Wave Phenomena, Academic Press, New York, 1984.

38 L. S. Schulman, Techniques and Applications of Path Integration, Wiley-Interscience, New York, 1981.

39 K. L. Baluja, P. G. Burke and L. A. Morgan, Comput. Phys. Commun., 1982, 27, 299.

40 A. Onsaki and H. Nakamura, Phys. Rev., 1990, 187, 1.

41 O. I. Tolstikhin, S. Watanabe and M. Matsuzawa, J. Phys. B: At. Mol. Opt. Phys., 1996, 29, L389.

42 O. I. Tolstikhin H. Nakamura, J. Chem. Phys., 1998, 108, 8899.

Paper 8/08551J 